\title{
Directed graph representation of half-rate additive codes over GF(4)
}

\author{
Lars Eirik Danielsen • Matthew G. Parker
}

Received: 10 February 2009 / Revised: 17 April 2009 / Accepted: 1 August 2010 /

Published online: 8 December 2010

(C) The Author(s) 2010. This article is published with open access at Springerlink.com

\begin{abstract}
We show that $\left(n, 2^{n}\right)$ additive codes over GF(4) can be represented as directed graphs. This generalizes earlier results on self-dual additive codes over GF(4), which correspond to undirected graphs. Graph representation reduces the complexity of code classification, and enables us to classify additive $\left(n, 2^{n}\right)$ codes over GF(4) of length up to 7 . From this we also derive classifications of isodual and formally self-dual codes. We introduce new constructions of circulant and bordered circulant directed graph codes, and show that these codes will always be isodual. A computer search of all such codes of length up to 26 reveals that these constructions produce many codes of high minimum distance. In particular, we find new near-extremal formally self-dual codes of length 11 and 13, and isodual codes of length 24, 25, and 26 with better minimum distance than the best known self-dual codes.
\end{abstract}

Keywords Additive codes · Quaternary codes · Classification · Graphs · Circulant codes · Formally self-dual codes

Mathematics Subject Classification (2000) 94B05 - 05C30

\section{Introduction}

An additive code, $\mathscr{C}$, over GF(4) of length $n$ is an additive subgroup of GF(4) ${ }^{n}$. We denote $\mathrm{GF}(4)=\left\{0,1, \omega, \omega^{2}\right\}$, where $\omega^{2}=\omega+1 . \mathscr{C}$ contains $2^{k}$ codewords for some $0 \leq k \leq 2 n$, and can be defined by a $k \times n$ generator matrix, with entries from GF(4), whose rows span $\mathscr{C}$ additively. $\mathscr{C}$ is called an $\left(n, 2^{k}\right)$ code. In this paper we will only consider $\left(n, 2^{n}\right)$, or half-rate, codes.

The Hamming weight of $\boldsymbol{u} \in \mathrm{GF}(4)^{n}$, denoted wt $(\boldsymbol{u})$, is the number of nonzero components of $\boldsymbol{u}$. The Hamming distance between $\boldsymbol{u}$ and $\boldsymbol{v}$ is $\operatorname{wt}(\boldsymbol{u}-\boldsymbol{v})$. The minimum distance of the code $\mathscr{C}$ is the minimal Hamming distance between any two distinct codewords of $\mathscr{C}$.

L. E. Danielsen $(\varangle) \cdot$ M. G. Parker

Department of Informatics, University of Bergen, PO Box 7803, 5020 Bergen, Norway

e-mail: larsed@ii.uib.no 
Since $\mathscr{C}$ is an additive code, the minimum distance is also given by the smallest nonzero weight of any codeword in $\mathscr{C}$. A code with minimum distance $d$ is called an $\left(n, 2^{k}, d\right)$ code. The weight distribution of the code $\mathscr{C}$ is the sequence $\left(A_{0}, A_{1}, \ldots, A_{n}\right)$, where $A_{i}$ is the number of codewords of weight $i$. The weight enumerator of $\mathscr{C}$ is the polynomial

$$
W_{\mathscr{C}}(x, y)=\sum_{i=0}^{n} A_{i} x^{n-i} y^{i}
$$

Two additive codes over GF(4), $\mathscr{C}$ and $\mathscr{C}^{\prime}$, are equivalent [9] if and only if the codewords of $\mathscr{C}$ can be mapped onto the codewords of $\mathscr{C}^{\prime}$ by a map that consists of a permutation of coordinates (or columns of the generator matrix), followed by multiplication of coordinates by nonzero elements from GF(4), followed by possible conjugation of coordinates. Conjugation of $x \in \mathrm{GF}(4)$ is defined by $\bar{x}=x^{2}$. For a code of length $n$, there is a total of $6^{n} n$ ! such maps. The six possible transformations given by scaling and conjugation of a coordinate are equivalent to the six permutations of the elements $\left\{1, \omega, \omega^{2}\right\}$ in the coordinate. A map that maps a code to itself is called an automorphism of the code. All automorphisms of $\mathscr{C}$ make up the automorphism group, denoted $\operatorname{Aut}(\mathscr{C})$. We can use the computational algebra system Magma [5] to find the automorphism group of a code. Since, at this time, Magma has no explicit function for calculating the automorphism group of an additive code, we use the following method, described by Calderbank et al. [4]. We map the $\left(n, 2^{k}\right)$ additive code $\mathscr{C}$ over $\mathrm{GF}(4)$ to the $[3 n, k]$ binary linear code $\beta(\mathscr{C})$ by applying the map $0 \mapsto 000$, $1 \mapsto 011, \omega \mapsto 101, \omega^{2} \mapsto 110$ to each generator of $\mathscr{C}$. We then use Magma to find $\operatorname{Aut}(\beta(\mathscr{C})) \cap \operatorname{Aut}\left(\beta\left(\mathrm{GF}(4)^{n}\right)\right)$, which will be isomorphic to $\operatorname{Aut}(\mathscr{C})$.

The trace map, $\operatorname{Tr}: \mathrm{GF}(4) \rightarrow \mathrm{GF}(2)$, is defined by $\operatorname{Tr}(x)=x+\bar{x}$. The Hermitian trace inner product of two vectors over GF(4) of length $n, \boldsymbol{u}=\left(u_{1}, u_{2}, \ldots, u_{n}\right)$ and $\boldsymbol{v}=$ $\left(v_{1}, v_{2}, \ldots, v_{n}\right)$, is given by

$$
\boldsymbol{u} * \boldsymbol{v}=\operatorname{Tr}(\boldsymbol{u} \cdot \overline{\boldsymbol{v}})=\sum_{i=1}^{n} \operatorname{Tr}\left(u_{i} \overline{v_{i}}\right)=\sum_{i=1}^{n}\left(u_{i} v_{i}^{2}+u_{i}^{2} v_{i}\right) \quad(\bmod 2)
$$

We define the dual of the code $\mathscr{C}$ with respect to the Hermitian trace inner product, $\mathscr{C}^{\perp}=$ $\left\{\boldsymbol{u} \in \mathrm{GF}(4)^{n} \mid \boldsymbol{u} * \boldsymbol{c}=0\right.$ for all $\left.\boldsymbol{c} \in \mathscr{C}\right\} . \mathscr{C}$ is self-dual if $\mathscr{C}=\mathscr{C}^{\perp}$, formally self-dual [13] if $W_{\mathscr{C}}(x, y)=W_{\mathscr{C} \perp}(x, y)$, and isodual if $\mathscr{C}$ is equivalent to $\mathscr{C}^{\perp}$. All self-dual codes are isodual, all isodual codes are formally self-dual, and all formally self-dual codes are half-rate codes. The set of linear half-rate codes over GF(4) is a small subset of the additive half-rate codes of even length. Optimal linear half-rate codes over GF(4) of length up to 18 were classified by Gulliver et al. [12]. The set of half-rate additive codes contains all self-dual, isodual, and formally self-dual additive codes, as well as all half-rate linear codes.

It follows from the Singleton bound [18] that any half-rate additive code over GF(4) must satisfy

$$
d \leq\left\lfloor\frac{n}{2}\right\rfloor+1 .
$$

$\mathscr{C}$ is called extremal if it attains the minimum distance $d$ given by the Singleton bound, and near-extremal if it has minimum distance $d-1$. If a code has highest possible minimum distance, but is not extremal, it is called optimal. Han and Kim [13,14] showed that there are no extremal formally self-dual codes of length $n \geq 8$, and no near-extremal formally self-dual codes of length $n=16, n=18$, or $n \geq 20$. Tighter bounds on the minimum distance of self-dual additive codes over GF(4) were given by Rains and Sloane [19, Theorem 33]. 
One of the motivations for studying self-dual additive codes over GF(4) has been the connection to quantum error-correcting codes [4]. Non-self-dual additive codes cannot be applied as quantum codes in the same way, but are interesting for other reasons. Han and Kim $[13,14]$ studied formally self-dual additive codes over GF(4), and showed that some of these codes have higher minimum distance than the best self-dual codes of the same length. Additive codes may also be better than the best linear codes of the same length. It is known that some strong binary codes can be projected onto additive codes over GF(4) [15]. A connection between formally self-dual codes over GF(4) and lattices has also been shown [6]. We have previously studied the connection between self-dual additive codes over $\operatorname{GF}\left(q^{2}\right)$, for any prime power $q$, and weighted graphs [7]. Such generalizations could also be considered for additive codes in general, but will not be discussed in this paper.

Let $t_{n}$ be the number of inequivalent codes of length $n$. To find one code from each of the $t_{n}$ equivalence classes, i.e., to classify the codes of length $n$, is a hard problem. We have previously classified all self-dual additive codes over GF(4) of length up to 12 [8], by using the fact that all such codes can be represented as undirected graphs [3,10,20,23], and that an operation called local complementation (LC) generates orbits of graphs that correspond to equivalence classes of codes [3,23].

The main result of this paper is to show that additive $\left(n, 2^{n}\right)$ codes over GF(4), except for some special cases, have representations as directed graphs. This basically transforms the problem of classifying such quaternary codes to a binary problem, with reduced complexity. We show that an algorithm by Östergård [17] for checking equivalence of linear codes also works for additive codes over GF(4). By using this algorithm, and the fact that codes correspond to directed graphs, we are able to classify additive $\left(n, 2^{n}\right)$ codes over GF(4) of length up to 7 . We find that there are more than two million inequivalent codes of length 7 . We have also checked which codes are formally self-dual, isodual, or self-dual, and give details of this enumeration. We introduce circulant and bordered circulant directed graph codes, and a computer search of all such codes up to length 26 reveals this subclass of additive half-rate codes to contain many codes with high minimum distance. Due to the structure of the generator matrices, codes from these constructions will always be isodual, and hence also formally self-dual. We construct new near-extremal formally self-dual codes of length 11 and 13, which were previously unknown [13]. This also answers in the affirmative the open question of the existence of an additive $\left(13,2^{13}, 6\right)$ code [1]. Finally, we find isodual codes of length 24,25 , and 26 with minimum distance 9 . The best known self-dual codes of these lengths have minimum distance 8 .

\section{Directed graph representation}

A directed graph is a pair $G=(V, E)$ where $V$ is a set of vertices, and $E \subseteq V \times V$ is a set of ordered pairs called edges. A graph with $n$ vertices can be represented by an $n \times n$ adjacency matrix $\Gamma$, where $\gamma_{i j}=1$ if $(i, j) \in E$, i.e., if there is a directed edge from $i$ to $j$, and $\gamma_{i j}=0$ otherwise. We will only consider simple graphs, where all diagonal elements of the adjacency matrix are 0 . The special case where we always have an edge $(j, i) \in E$ whenever there is an edge $(i, j) \in E$, i.e., the adjacency matrix is symmetric, is called an undirected graph. The in-neighbourhood of $v \in V$, denoted $\mathrm{NI}_{v} \subset V$, is the set of vertices $i$ such that there is a directed edge $(i, v) \in E$. Similarly, $\mathrm{NO}_{v} \subset V$ is the out-neighbourhood of $v$, i.e., the set of vertices $i$ such that there is a directed edge $(v, i)$ in $E$. $\left|\mathrm{NI}_{v}\right|$ is the indegree of $v$, and $\left|\mathrm{NO}_{v}\right|$ is the outdegree of $v$. Two graphs $G=(V, E)$ and $G^{\prime}=\left(V, E^{\prime}\right)$ are isomorphic if and only if there exists a permutation $\pi$ of $V$ such that $(u, v) \in E \Longleftrightarrow(\pi(u), \pi(v)) \in E^{\prime}$. 
A directed graph is connected, (also known as weakly connected), if we can reach any vertex starting from any other vertex by traversing edges in some direction, i.e., not necessarily in the direction they point.

Definition 1 A directed graph code is an additive $\left(n, 2^{n}\right)$ code over GF(4) that has a generator matrix of the form $C=\Gamma+\omega I$, where $\Gamma$ is the adjacency matrix of a simple directed graph and $I$ is the identity matrix.

Proposition 1 Given a directed graph code $\mathscr{C}$ with generator matrix $C=\Gamma+\omega I$, its dual $\mathscr{C}^{\perp}$ is generated by $C^{T}$.

Proof We must show that for any $c \in \mathscr{C}$ and any $c^{\prime} \in \mathscr{C}^{\perp}$, the trace inner product $c * c^{\prime}=$ $\operatorname{Tr}\left(c \cdot \overline{c^{\prime}}\right)=0$. Let $c=a C$ and $c^{\prime}=b C^{T}$, with $a, b \in \mathrm{GF}(2)^{n}$. Then $c * c^{\prime}=(a C) *\left(b C^{T}\right)=$ $\operatorname{Tr}\left((a C) \cdot \overline{\left(b C^{T}\right)}\right)=\operatorname{Tr}\left((a C)\left(b \bar{C}^{T}\right)^{T}\right)=\operatorname{Tr}\left(a C \bar{C} b^{T}\right)$, which must be 0 if all elements of $C \bar{C}$ are from GF(2). This is clearly the case, since $C \bar{C}=(\Gamma+\omega I)\left(\Gamma+\omega^{2} I\right)=\Gamma^{2}+\Gamma+I$.

Theorem 1 Given an additive $\left(n, 2^{n}\right)$ code $\mathscr{C}$ over $\mathrm{GF}(4)$ with generator matrix $C$, there exists a directed graph code equivalent to $\mathscr{C}$, except when $C=A+\omega B$ is such that all $2^{n}$ sets of $n$ columns $\left(\left\{\boldsymbol{a}_{1}, \boldsymbol{b}_{1}\right\},\left\{\boldsymbol{a}_{2}, \boldsymbol{b}_{2}\right\}, \ldots,\left\{\boldsymbol{a}_{n}, \boldsymbol{b}_{n}\right\}\right)$ of $(A \mid B)$, where $\left\{\boldsymbol{a}_{i}, \boldsymbol{b}_{i}\right\}$ means that we choose either $\boldsymbol{a}_{i}$ or $\boldsymbol{b}_{i}$, are linearly dependent.

Proof We can write $C=A+\omega B$, with $(A \mid B)$ a binary $n \times 2 n$ matrix. From the fact that the rows of $C$ additively span a vector space of dimension $n$, it follows that $(A \mid B)$ has full rank. If the $n \times n$ submatrix $B$ also has full rank over $\mathrm{GF}(2)$, we simply perform the basis change $B^{-1}(A \mid B)=\left(\Gamma^{\prime} \mid I\right)$. Any non-zero elements on the diagonal of $\Gamma^{\prime}$ can simply be set to zero, effected by conjugating the corresponding coordinates of $\Gamma^{\prime}+\omega I$, to obtain an equivalent directed graph code generated by $\Gamma+\omega I$.

In the case that $B$ does not have full rank, we must show that there is a code $\mathscr{C}^{\prime}$, equivalent to $\mathscr{C}$, generated by $A^{\prime}+\omega B^{\prime}$ where $B^{\prime}$ does have full rank. Then we can apply the method described in the first part of this proof to obtain the graph form. Let the columns of $A$ be denoted $\left(\boldsymbol{a}_{1}, \boldsymbol{a}_{2}, \ldots, \boldsymbol{a}_{n}\right)$ and the columns of $B$ be denoted $\left(\boldsymbol{b}_{1}, \boldsymbol{b}_{2}, \ldots, \boldsymbol{b}_{n}\right)$. Observe that multiplying column $i$ of $C$ by $\omega^{2}$, followed by conjugation of the same column, has the effect of swapping columns $\boldsymbol{a}_{i}$ and $\boldsymbol{b}_{i}$ in $(A \mid B)$. Since we know that at least one of the $2^{n}$ possible choices $\left(\left\{\boldsymbol{a}_{1}, \boldsymbol{b}_{1}\right\},\left\{\boldsymbol{a}_{2}, \boldsymbol{b}_{2}\right\}, \ldots,\left\{\boldsymbol{a}_{n}, \boldsymbol{b}_{n}\right\}\right)$ span a vector space of dimension $n$, we can find a matrix $\left(A^{\prime} \mid B^{\prime}\right)$ where $B^{\prime}$ has full rank.

It follows from Proposition 1 that a directed graph code is self-dual if and only if its generator matrix is symmetric, i.e., it is in fact an undirected graph code. The fact that all self-dual additive codes over GF(4) can be represented as undirected graphs is well known [3,10,20,23], and was used to classify all self-dual additive codes up to length 12 [8]. Theorem 1 is a generalization of this result to the much larger classes of directed graphs and half-rate additive codes over GF(4). As stated in Theorem 1, some special codes do not have graph representations. These are codes that will typically not be of interest, such as codes with a generator matrix that contains an all-zero column or a set of linearly dependent binary columns (up to scaling by $\omega$ or $\omega^{2}$ ). 
Example 1 We consider an additive $\left(7,2^{7}, 4\right)$ code, $\mathscr{C}$, generated by

$$
\begin{gathered}
C=\left(\begin{array}{ccccccc}
\omega^{2} & 0 & 0 & 1 & 0 & \omega & \omega \\
1 & 0 & 0 & 0 & 1 & 1 & 1 \\
0 & 0 & \omega & 0 & \omega^{2} & \omega^{2} & \omega \\
0 & 0 & 1 & 1 & \omega^{2} & \omega & \omega^{2} \\
1 & \omega & 0 & 1 & \omega^{2} & \omega^{2} & 0 \\
1 & 1 & 1 & 1 & \omega^{2} & 1 & 1 \\
0 & 0 & 1 & \omega & 1 & \omega^{2} & 1
\end{array}\right)=A+\omega \\
(A \mid B)=\left(\begin{array}{lllllll|lllllll}
1 & 0 & 0 & 1 & 0 & 0 & 0 & 1 & 0 & 0 & 0 & 0 & 1 & 1 \\
1 & 0 & 0 & 0 & 1 & 1 & 1 & 0 & 0 & 0 & 0 & 0 & 0 & 0 \\
0 & 0 & 0 & 0 & 1 & 1 & 0 & 0 & 0 & 1 & 0 & 1 & 1 & 1 \\
0 & 0 & 1 & 1 & 1 & 0 & 1 & 0 & 0 & 0 & 0 & 1 & 1 & 1 \\
1 & 0 & 0 & 1 & 1 & 1 & 0 & 0 & 1 & 0 & 0 & 1 & 1 & 0 \\
1 & 1 & 1 & 1 & 1 & 1 & 1 & 0 & 0 & 0 & 0 & 1 & 0 & 0 \\
0 & 0 & 1 & 0 & 1 & 1 & 1 & 0 & 0 & 0 & 1 & 0 & 1 & 0
\end{array}\right) .
\end{gathered}
$$

We swap column $a_{6}$ with $b_{6}$ and column $a_{7}$ with $b_{7}$ to get the matrix

$$
\left(A^{\prime} \mid B^{\prime}\right)=\left(\begin{array}{lllllll|lllllll}
1 & 0 & 0 & 1 & 0 & 1 & 1 & 1 & 0 & 0 & 0 & 0 & 0 & 0 \\
1 & 0 & 0 & 0 & 1 & 0 & 0 & 0 & 0 & 0 & 0 & 0 & 1 & 1 \\
0 & 0 & 0 & 0 & 1 & 1 & 1 & 0 & 0 & 1 & 0 & 1 & 1 & 0 \\
0 & 0 & 1 & 1 & 1 & 1 & 1 & 0 & 0 & 0 & 0 & 1 & 0 & 1 \\
1 & 0 & 0 & 1 & 1 & 1 & 0 & 0 & 1 & 0 & 0 & 1 & 1 & 0 \\
1 & 1 & 1 & 1 & 1 & 0 & 0 & 0 & 0 & 0 & 0 & 1 & 1 & 1 \\
0 & 0 & 1 & 0 & 1 & 1 & 0 & 0 & 0 & 0 & 1 & 0 & 1 & 1
\end{array}\right)
$$

where $B^{\prime}$ has full rank. We can then obtain the matrix

$$
\left(\Gamma^{\prime} \mid I\right)=B^{\prime-1}\left(A^{\prime} \mid B^{\prime}\right)=\left(\begin{array}{lllllll|lllllll}
1 & 0 & 0 & 1 & 0 & 1 & 1 & 1 & 0 & 0 & 0 & 0 & 0 & 0 \\
0 & 0 & 1 & 0 & 1 & 0 & 1 & 0 & 1 & 0 & 0 & 0 & 0 & 0 \\
1 & 0 & 1 & 1 & 1 & 0 & 0 & 0 & 0 & 1 & 0 & 0 & 0 & 0 \\
1 & 0 & 1 & 0 & 0 & 1 & 0 & 0 & 0 & 0 & 1 & 0 & 0 & 0 \\
0 & 1 & 1 & 1 & 0 & 0 & 0 & 0 & 0 & 0 & 0 & 1 & 0 & 0 \\
1 & 1 & 0 & 0 & 0 & 1 & 1 & 0 & 0 & 0 & 0 & 0 & 1 & 0 \\
0 & 1 & 0 & 0 & 1 & 1 & 1 & 0 & 0 & 0 & 0 & 0 & 0 & 1
\end{array}\right) .
$$

By setting the diagonal of $\Gamma^{\prime}$ to zero, we get the adjacency matrix of a simple directed graph,

$$
\Gamma=\left(\begin{array}{lllllll}
0 & 0 & 0 & 1 & 0 & 1 & 1 \\
0 & 0 & 1 & 0 & 1 & 0 & 1 \\
1 & 0 & 0 & 1 & 1 & 0 & 0 \\
1 & 0 & 1 & 0 & 0 & 1 & 0 \\
0 & 1 & 1 & 1 & 0 & 0 & 0 \\
1 & 1 & 0 & 0 & 0 & 0 & 1 \\
0 & 1 & 0 & 0 & 1 & 1 & 0
\end{array}\right)
$$

This graph is shown in Fig. $1 . \Gamma+\omega I$ generates a $\left(7,2^{7}, 4\right)$ directed graph code equivalent to $\mathscr{C}$. 
Fig. 1 Directed graph representation of a $\left(7,2^{7}, 4\right)$ code

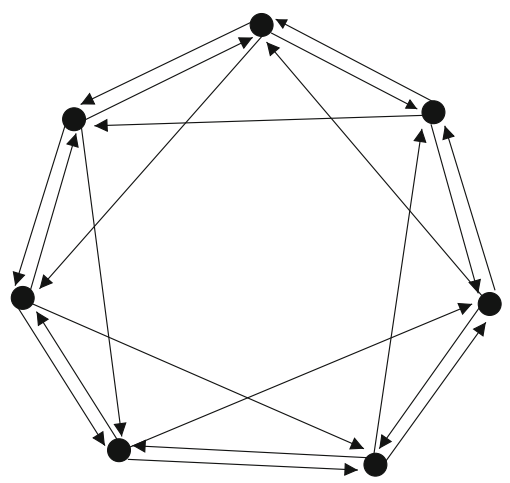

\section{Classification}

Since we have shown in Theorem 1 that, except for some special cases, additive codes over GF(4) are equivalent to directed graph codes, it follows that to classify such codes, we only need to consider directed graphs. Furthermore, we only need to consider non-isomorphic graphs, since if two directed graphs are isomorphic, the corresponding directed graph codes are equivalent by a permutation of coordinates. All non-isomorphic connected directed graphs on up to 7 vertices can be generated in a few hours by using tools provided with the software package nauty [16]. For an enumeration of these graphs, see sequence A003085 in The On-Line Encyclopedia of Integer Sequences [21].

Connected graphs correspond to indecomposable codes. A code is decomposable if it can be written as the direct sum of two smaller codes. For example, let $\mathscr{C}$ be an $\left(n, 2^{n}, d\right)$ code and $\mathscr{C}^{\prime}$ an $\left(n^{\prime}, 2^{n^{\prime}}, d^{\prime}\right)$ code. The direct sum, $\mathscr{C} \oplus \mathscr{C}^{\prime}=\left\{u \| v \mid u \in \mathscr{C}, v \in \mathscr{C}^{\prime}\right\}$, where $\|$ means concatenation, is an $\left(n+n^{\prime}, 2^{n+n^{\prime}}, \min \left\{d, d^{\prime}\right\}\right)$ code. It follows that all decomposable codes of length $n$ can be classified easily once all indecomposable codes of length less than $n$ are known. The total number of codes of length $n, t_{n}$, is easily derived from the numbers $i_{n}$ of indecomposable codes by using the Euler transform [22],

$$
\begin{aligned}
c_{n} & =\sum_{d \mid n} d i_{d} \\
t_{1} & =c_{1} \\
t_{n} & =\frac{1}{n}\left(c_{n}+\sum_{k=1}^{n-1} c_{k} t_{n-k}\right) .
\end{aligned}
$$

To check whether two additive codes over GF(4) are equivalent, we use a modified version of an algorithm originally devised by Östergård [17] for checking equivalence of linear codes. We show that this method also works for additive codes. An additive code over GF(4) is mapped to an undirected colored code graph in the following way. (Note that this representation is not related to the directed graph representation defined previously.) First, we find a set of vectors of some weights that generate the code. Often, the set of all vectors of minimum weight $d$ will suffice, otherwise, we add all vectors of weight $d+1$, and then all vectors of weight $d+2, \ldots$, as necessary. For each vector $c_{i}$ in the resulting set, add a vertex $v_{i}$ to the code graph. Also add $n$ sets of three vertices, where $n$ is the length of the code. Each set of three vertices represent the non-zero elements $\left\{1, \omega, \omega^{2}\right\} \in \mathrm{GF}(4)$ in one coordinate. In every set, each of the three vertices is connected to each of the two other by undirected 
Fig. 2 Example of code graph for checking equivalence

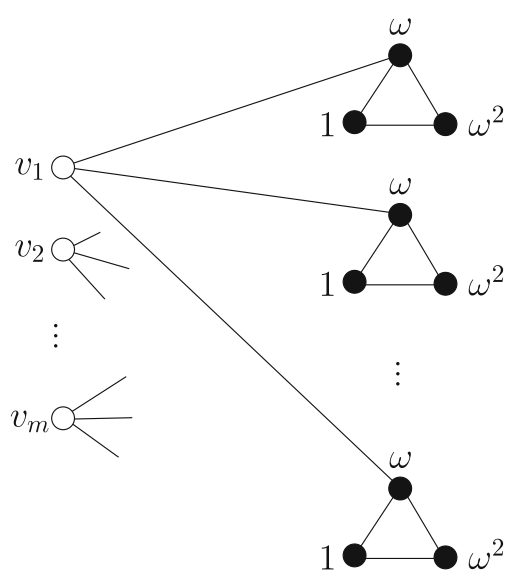

edges, to form a cycle. (This corresponds to the fact that any permutation of the symbols $\left\{1, \omega, \omega^{2}\right\}$ in each coordinate of the code gives an equivalent code.) Let the vertices $v_{i}$ have one color, and the $3 n$ other vertices have a different color. Add edges between vertex $v_{i}$ and the $n 3$-cycles corresponding to the codeword $c_{i}$. E.g., if $c_{i}$ has $\omega$ in coordinate $j$, then there is an edge between $v_{i}$ and the element labelled $\omega$ in the $j$ th 3-cycle. As an example, Fig. 2 shows the case where $c_{1}=(\omega, \omega, \ldots, \omega)$. The resulting code graph is then canonized, i.e., relabelled, but with coloring preserved, using the nauty software [16]. If two graphs are isomorphic, their canonical representations are guaranteed to be the same. Hence, if two codes are equivalent, their canonical code graphs will be identical. Furthermore, as an alternative to the method described in the introduction, we can find the automorphism group of a code as the automorphism group of its code graph, i.e., the set of all vertex permutations that map the code graph to itself.

To classify codes of length up to 7, we take all non-isomorphic connected directed graphs, map them to codes, and canonize the corresponding code graphs as described above. All duplicates are removed to obtain one representative from each equivalence class. The special form of the generator matrix of a directed graph code makes it easier to find all codewords of small weight. If $\mathscr{C}$ is generated by $C=\Gamma+\omega I$, then any codeword formed by adding $i$ rows of $C$ must have weight at least $i$. This means that we can find all codewords of weight $i$ by only considering sums of at most $i$ rows of $C$. This property also helps when we want to find the minimum distance of a code. Furthermore, if we wanted to exclude codes with minimum distance one from our classification, it would suffice to exclude graphs where some vertex has outdegree zero, since this would imply that there is a row in the generator matrix with weight one.

We also note another special property of directed graph codes: Given a directed graph code, $\mathscr{C}$, with generator matrix $\Gamma+\omega I$, it can be verified that the additive code over $\mathbb{Z}_{4}$ given by $2 \Gamma+I$ always has the same weight distribution as $\mathscr{C}$. We may therefore replace the elements from GF(4) with elements from $\mathbb{Z}_{4}$ by the mapping $0 \mapsto 0,1 \mapsto 2, \omega \mapsto 1, \omega^{2} \mapsto 3$.

Table 1 gives the number of half-rate additive codes over GF(4) by length and minimum distance. Note that only indecomposable codes are counted, and that the special cases in Theorem 1, that do not have a directed graph representation, are not included. A database containing one representative from each equivalence class is available at http://www.ii.uib. no/ larsed/directed/. Tables 2 and 3 give the numbers of formally self-dual and isodual codes. (Note that the 240 formally self-dual $\left(6,2^{6}, 3\right)$-codes and 3 formally self-dual $\left(7,2^{7}, 4\right)$-codes 
Table 1 Number of half-rate additive codes over GF(4)

Table 2 Number of formally self-dual additive codes over $\mathrm{GF}(4)$

Table 3 Number of isodual additive codes over GF(4)

Table 4 Number of self-dual additive codes over GF(4)

\begin{tabular}{llrrrll}
\hline$d \backslash n$ & 2 & 3 & 4 & 5 & 6 & 7 \\
\hline 1 & 1 & 4 & 27 & 322 & 8,509 & 686,531 \\
2 & 1 & 3 & 21 & 262 & 9,653 & $1,279,641$ \\
3 & & & 1 & 9 & 644 & 253,635 \\
4 & & & & & 1 & 3 \\
Total & 2 & 7 & 49 & 593 & 18,807 & $2,219,810$ \\
\hline
\end{tabular}

\begin{tabular}{llrrrll}
\hline$d \backslash n$ & 2 & 3 & 4 & 5 & 6 & 7 \\
\hline 1 & 1 & 1 & 10 & 55 & 1,082 & 36,129 \\
2 & 1 & 2 & 12 & 79 & 2,348 & 192,201 \\
3 & & & 1 & 5 & 240 & 55,711 \\
4 & & & & & 1 & 3 \\
Total & 2 & 3 & 23 & 139 & 3,671 & 284,044 \\
\hline
\end{tabular}

\begin{tabular}{lrrrrll}
\hline$d \backslash n$ & 2 & 3 & \multicolumn{1}{c}{4} & 5 & 6 & 7 \\
\hline 1 & 1 & 1 & 8 & 27 & 344 & 3,243 \\
2 & 1 & 2 & 10 & 45 & 598 & 8517 \\
3 & & & 1 & 5 & 124 & 3,299 \\
4 & & & & & 1 & 3 \\
Total & 2 & 3 & 19 & 77 & 1,067 & 15,062 \\
\hline
\end{tabular}

\begin{tabular}{lllllrr}
\hline$d \backslash n$ & 2 & 3 & 4 & 5 & 6 & 7 \\
\hline 2 & 1 & 1 & 2 & 3 & 9 & 22 \\
3 & & & & 1 & 1 & 4 \\
4 & 1 & 1 & 2 & 4 & 11 & 26 \\
Total & 1 & & & & 1 & \\
\hline
\end{tabular}

were also found by Han and Kim [13].) For completeness, we include in Table 4 the number of self-dual codes, although we have previously classified these up to length 12 [8].

\section{Circulant directed graph codes}

Since it is infeasible to study all half-rate additive codes of lengths much higher than those classified in the previous section, we restrict our search space to codes corresponding to graphs with circulant adjacency matrices. A matrix is circulant if the $i$ th row is equal to the first row, cyclically shifted $i-1$ times to the right. The generator matrix of a directed graph code is obtained by setting all diagonal elements of the circulant adjacency matrix to $\omega$. There are $2^{n-1}$ such codes of length $n$, some of which may be equivalent. We also consider 
Table 5 Number of circulant and bordered circulant directed graph codes of highest found minimum distance

\begin{tabular}{|c|c|c|c|}
\hline$n$ & $\operatorname{Max} d$ & \# Codes & \# Self-dual \\
\hline 2 & 2 & 1 & 1 \\
\hline 3 & 2 & 2 & 1 \\
\hline 4 & 3 & 1 & 0 \\
\hline 5 & 3 & 3 & 1 \\
\hline 6 & 4 & 1 & 1 \\
\hline 7 & 4 & 2 & 0 \\
\hline 8 & 4 & 11 & 1 \\
\hline 9 & 4 & 22 & 2 \\
\hline 10 & 5 & 4 & 0 \\
\hline 11 & 5 & 21 & 0 \\
\hline 12 & 6 & 2 & 1 \\
\hline 13 & 6 & 2 & 0 \\
\hline 14 & 6 & 54 & 3 \\
\hline 15 & 6 & 325 & 3 \\
\hline 16 & 7 & 1 & 0 \\
\hline 17 & 7 & 9 & 1 \\
\hline 18 & 8 & 1 & 1 \\
\hline 19 & 7 & 1,366 & 4 \\
\hline 20 & 8 & 4 & 3 \\
\hline 21 & 8 & 42 & 0 \\
\hline 22 & 8 & 1,328 & 17 \\
\hline 23 & 8 & 8,027 & 2 \\
\hline 24 & 9 & 1 & 0 \\
\hline 25 & 9 & 25 & 0 \\
\hline 26 & 9 & 1,877 & 0 \\
\hline
\end{tabular}

bordered circulant adjacency matrices: Given a length $n$ circulant graph code with generator matrix $C$, we obtain a code of length $n+1$ with generator matrix

$$
\left(\begin{array}{llll}
\omega & 1 & \cdots & 1 \\
1 & & \\
\vdots & C & \\
1 & &
\end{array}\right) .
$$

There are $2^{n-2}$ such codes of length $n$, some of which may be equivalent. For each $n$ up to 26 , we have counted, up to equivalence, all circulant and bordered circulant directed graph codes. The number of codes of the highest found minimum distance for each $n$ is given in Table 5. A database of these codes is available at http://www.ii.uib.no/ larsed/directed/.

Proposition 2 A circulant or bordered circulant directed graph code will always be isodual.

Proof A circulant directed graph code of length $n$ has generator matrix 


$$
C=\left(\begin{array}{lllll}
\omega & a_{1} & a_{2} & \cdots & a_{n} \\
a_{n} & \omega & a_{1} & \cdots & a_{n-1} \\
a_{n-1} & a_{n} & \omega & \cdots & a_{n-2} \\
\vdots & \vdots & \vdots & \ddots & \vdots \\
a_{1} & a_{2} & a_{3} & \cdots & \omega
\end{array}\right),
$$

where $\left(a_{1}, a_{2}, \ldots, a_{n}\right)$ is any binary sequence of length $n-1$. It follows from Proposition 1 that the dual code is generated by

$$
C^{T}=\left(\begin{array}{lllll}
\omega & a_{n} & a_{n-1} & \cdots & a_{1} \\
a_{1} & \omega & a_{n} & \cdots & a_{2} \\
a_{2} & a_{1} & \omega & \cdots & a_{3} \\
\vdots & \vdots & \vdots & \ddots & \vdots \\
a_{n} & a_{n-1} & a_{n-2} & \cdots & \omega
\end{array}\right) .
$$

We can obtain $C^{T}$ from $C$ by reversing the order of the columns, and then reversing the order of the rows. Permuting rows has no effect on the code, and permuting columns produces an equivalent code. Hence the code must be equivalent to its dual. The same argument holds for bordered circulant codes, except that the first row and column remain fixed.

If $\mathscr{C}$ is a circulant directed graph code of length $n$, then $|\operatorname{Aut}(\mathscr{C})|$ must be divisible by $n$, since the structure of the generator matrix ensures that cyclically shifting all codewords will preserve the code. Similarly, if $\mathscr{C}$ is a bordered circulant directed graph code of length $n$, then $|\operatorname{Aut}(\mathscr{C})|$ must be divisible by $n-1$, since the code is preserved by fixing the first coordinate and cyclically shifting the last $n-1$ coordinates.

With our method, we are able to find new codes, since the existence of near-extremal formally self-dual codes of lengths 11 and 13 was previously an open problem [13]. We also answer the question of the existence of an additive $\left(13,2^{13}, 6\right)$ code in the positive. Parameters for optimal additive codes over GF(4) were determined for $n \leq 12$ by Blockhuis and Brouwer [2], and for $n \leq 13$ by Bierbrauer et al. [1], by using geometric descriptions of codes. Bierbrauer et al. found an additive $\left(11,2^{11}, 5\right)$ code, but posed as an open question the existence of an additive $\left(13,2^{13}, 6\right)$ code.

There are at least 21 formally self-dual $\left(11,2^{11}, 5\right)$ codes, with generators available at http://www.ii.uib.no/ larsed/directed/. We find codes with five different weight enumerators:

$$
\begin{aligned}
& W_{11,1}(1, y)=1+55 y^{5}+242 y^{6}+275 y^{7}+495 y^{8}+605 y^{9}+286 y^{10}+89 y^{11}, \\
& W_{11,2}(1, y)=1+66 y^{5}+198 y^{6}+330 y^{7}+495 y^{8}+550 y^{9}+330 y^{10}+78 y^{11}, \\
& W_{11,3}(1, y)=1+70 y^{5}+182 y^{6}+350 y^{7}+495 y^{8}+530 y^{9}+346 y^{10}+74 y^{11}, \\
& W_{11,4}(1, y)=1+75 y^{5}+162 y^{6}+375 y^{7}+495 y^{8}+505 y^{9}+366 y^{10}+69 y^{11}, \\
& W_{11,5}(1, y)=1+77 y^{5}+154 y^{6}+385 y^{7}+495 y^{8}+495 y^{9}+374 y^{10}+67 y^{11}
\end{aligned}, .
$$

There are five codes with weight enumerator $W_{11,1}$. Of these, three have automorphism groups of order 11, one has 10 automorphisms, and one has 110 automorphisms. There are four codes with $W_{11,2}$, all with 11 automorphisms. There are two codes with $W_{11,3}$, both with 10 automorphisms. There are five codes with $W_{11,4}$, all with 10 automorphisms. There are five codes with $W_{11,5}$, all with 11 automorphisms.

We have found two formally self-dual $\left(13,2^{13}, 6\right)$ codes. $\mathscr{C}_{13,1}$ is generated by all cyclic shifts of $(\omega 101001110000)$ and has an automorphism group of order $13 . \mathscr{C}_{13,2}$ is generated 
by all cyclic shifts of $(\omega 111011111010)$ and has an automorphism group of order 78 . Both these codes have the same weight enumerator:

$$
\begin{aligned}
W_{13}(1, y)=1 & +247 y^{6}+481 y^{7}+936 y^{8}+1625 y^{9}+2197 y^{10}+1755 y^{11} \\
& +715 y^{12}+235 y^{13}
\end{aligned}
$$

Note that for several lengths, there are no self-dual codes among the circulant and bordered circulant codes with highest minimum distance. The best known self-dual codes of length 24,25 , and 26 have minimum distance 8 . We find a single isodual $\left(24,2^{24}, 9\right)$ code generated by the cyclic shifts of $(\omega 01101111111111010000110)$ with automorphism group of order 72 and weight enumerator

$$
\begin{aligned}
W_{24}(1, y)=1 & +1752 y^{9}+8748 y^{10}+26064 y^{11}+81408 y^{12}+232776 y^{13} \\
& +573516 y^{14}+1119264 y^{15}+1869777 y^{16}+2676456 y^{17}+3096804 y^{18} \\
& +2959056 y^{19}+2204568 y^{20}+1255416 y^{21}+520740 y^{22}+134208 y^{23} \\
& +16662 y^{24}
\end{aligned}
$$

We also find 25 isodual $\left(25,2^{25}, 9\right)$ codes with 25 different weight enumerators, and 1877 isodual $\left(26,2^{26}, 9\right)$ codes with 1865 different weight enumerators.

We have previously studied circulant undirected graph codes [7]. There are only $2^{\left\lceil\frac{n-1}{2}\right\rceil}$ such codes of length $n$, due to the fact that the generator matrix must be symmetric. Gulliver and Kim [11] also performed a computer search of circulant self-dual additive codes over $\mathrm{GF}(4)$, but their search was not restricted to graph codes.

A particularly interesting type of circulant code is a type of quadratic residue code [18]. The length of such a code must be a prime $p$. When $p \equiv 1(\bmod 4)$, the quadratic residue code will be self-dual, and the corresponding undirected graph is known as a Paley graph. When $p \equiv 3(\bmod 4)$, the code will only be isodual. The first row of the generator matrix of the code is $\left(\omega, l_{1}, \ldots, l_{p-1}\right)$, where $l_{i}=1$ if $i$ is a quadratic residue modulo $p$, i.e., if $x^{2} \equiv i$ $(\bmod p)$ has a solution $x \in \mathrm{GF}(p)$. Otherwise, $l_{i}=0$. Many codes with high minimum distance can be obtained from this construction. For instance, by bordering quadratic residue codes, as described above, we obtain self-dual $\left(6,2^{6}, 4\right),\left(14,2^{14}, 6\right)$, and $\left(30,2^{30}, 12\right)$ codes, and isodual $\left(4,2^{4}, 3\right),\left(8,2^{8}, 4\right)$, and $\left(12,2^{12}, 6\right)$ codes.

Acknowledgments The authors would like to thank Jürgen Bierbrauer for helpful comments. This research was supported by the Research Council of Norway.

Open Access This article is distributed under the terms of the Creative Commons Attribution Noncommercial License which permits any noncommercial use, distribution, and reproduction in any medium, provided the original author(s) and source are credited.

\section{References}

1. Bierbrauer J., Edel Y., Faina G., Marcugini S., Pambianco F.: Short additive quaternary codes. IEEE Trans. Inf. Theory 55(3), 952-954 (2009).

2. Blokhuis A., Brouwer A.E.: Small additive quaternary codes. Eur. J. Combin. 25(2), 161-167 (2004).

3. Bouchet A.: Graphic presentations of isotropic systems. J. Combin. Theory Ser. B 45(1), 58-76 (1988).

4. Calderbank A.R., Rains E.M., Shor P.M., Sloane N.J.A.: Quantum error correction via codes over GF(4). IEEE Trans. Inf. Theory 44(4), 1369-1387 (1998).

5. Cannon J., Bosma W.: Handbook of Magma Functions, Version 2.14. http://magma.maths.usyd.edu.au/ (2007). 
6. Choie Y., Solé P.: Broué-Enguehard maps and Atkin-Lehner involutions. Eur. J. Combin. 29(1), 24-34 (2008).

7. Danielsen L.E.: Graph-based classification of self-dual additive codes over finite fields. Adv. Math. Commun. 3(4), 329-348 (2009).

8. Danielsen L.E., Parker M.G.: On the classification of all self-dual additive codes over GF(4) of length up to 12. J. Combin. Theory Ser. A 113(7), 1351-1367 (2006).

9. Gaborit P., Huffman W.C., Kim J.L., Pless V.: On additive GF(4) codes. In: Codes and Association Schemes. DIMACS Series in Discrete Mathematics and Theoretical Computer Science, vol. 56, pp. 135149. American Mathematical Society, Providence, RI (2001)

10. Grassl M., Klappenecker A., Rötteler M.: Graphs, quadratic forms, and quantum codes. In: Proceedings of IEEE International Symposium on Information Theory, p. 45 (2002).

11. Gulliver T.A., Kim J.L.: Circulant based extremal additive self-dual codes over GF(4). IEEE Trans. Inform. Theory 50(2), 359-366 (2004).

12. Gulliver T.A., Östergård P.R.J., Senkevitch N.I.: Optimal quaternary linear rate-1/2 codes of length $\leq 18$. IEEE Trans. Inform. Theory 49(6), 1540-1543 (2003).

13. Han S., Kim J.L.: Formally self-dual additive codes over $\mathbb{F}_{4}$. J. Sym. Comput. 45(7), 787-799 (2010).

14. Han, S., Kim, J.L.: The nonexistence of near-extremal formally self-dual codes. Des. Codes Cryptogr. 51(1), 69-77 (2009)

15. Kim J.L., Mellinger K.E., Pless V.: Projections of binary linear codes onto larger fields. SIAM J. Discret. Math. 16(4), 591-603 (2003).

16. McKay B.D.: Nauty User's Guide, Version 2.2. http://cs.anu.edu.au/ bdm/nauty/ (2004).

17. Östergård P.R.J.: Classifying subspaces of Hamming spaces. Des. Codes Cryptogr. 27(3), 297-305 (2002).

18. Pless V.S., Huffman W.C. (eds.): Handbook of Coding Theory. North-Holland, Amsterdam (1998).

19. Rains E.M., Sloane N.J.A.: Self-dual codes. In: Handbook of Coding Theory, pp. 177-294. North-Holland, Amsterdam (1998).

20. Schlingemann D., Werner R.F.: Quantum error-correcting codes associated with graphs. Phys. Rev. A 65(1), 012308 (2002).

21. Sloane N.J.A.: The On-Line Encyclopedia of Integer Sequences. http://www.research.att.com/ njas/ sequences/ (2010).

22. Sloane N.J.A., Plouffe S.: The Encyclopedia of Integer Sequences. Academic Press, San Diego, CA (1995).

23. Van den Nest M., Dehaene J., De Moor B.: Graphical description of the action of local Clifford transformations on graph states. Phys. Rev. A 69(2), 022316 (2004). 